\title{
Spinal cord injured women treated with Lokomat(B) Robot Considerations for improved treatment effectiveness
}

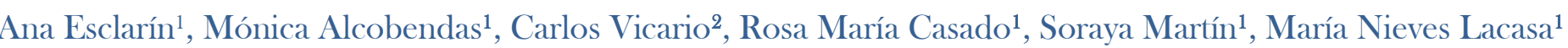

${ }^{1}$ Department of Physical Medicine and Rehabilitation. Hospital Nacional Paraplejicos. Toledo. Spain. ${ }^{2}$ Department of Orthopedic Surgery. Hospital Nuestra Senora del Prado. Talavera de la Reina. Spain

\section{Introduction.-}
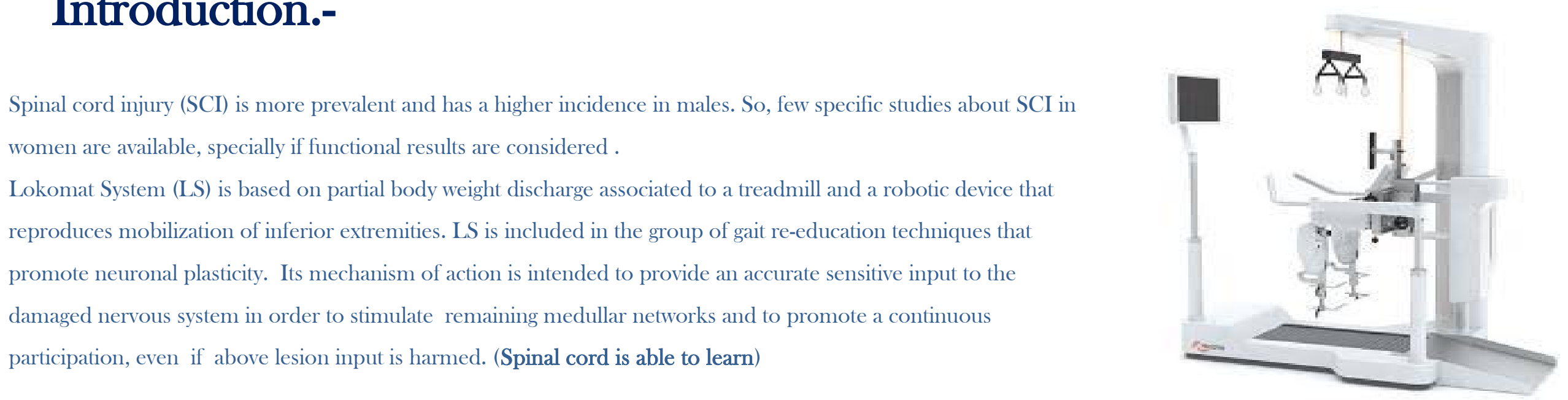

Purpose.-

To know functional and epidemiologic characteristics of women that have undergone treatment program with Lokomat System ${ }^{\circledR}$ and to determinate the best moment for this therapy.

Method.-

Exclusion criteria

Complete SCI / More than 6 months of evolution / Unstable fracture / Severe bronchial or cardiac pathology / Severe osteoporosis / Pressure ulcers / Arthropaty or flexion deformities / Dismetry $>2 \mathrm{~cm} /$ Weight $>150 \mathrm{~kg}$ or Height $>2 \mathrm{~m}$
Intervention

Forty 30-minute sessions, 5 days per week, beginning with a discharge of $60 \%$ of body weight and in a comfortable speed for the patient.

\section{Outcome measures:}

Demographic and lesion data / Lower extremity muscle strength (LEMS) / SCIM II (Spinal cord independence measure) / FIM (Functional independence measure) WISCI II (Walking Index Spinal Cord Injury) Test 10m / Test 6 ${ }^{\prime}$ / Up and Go EuroQol 5D (percepción de calidad de vida)
Results.-

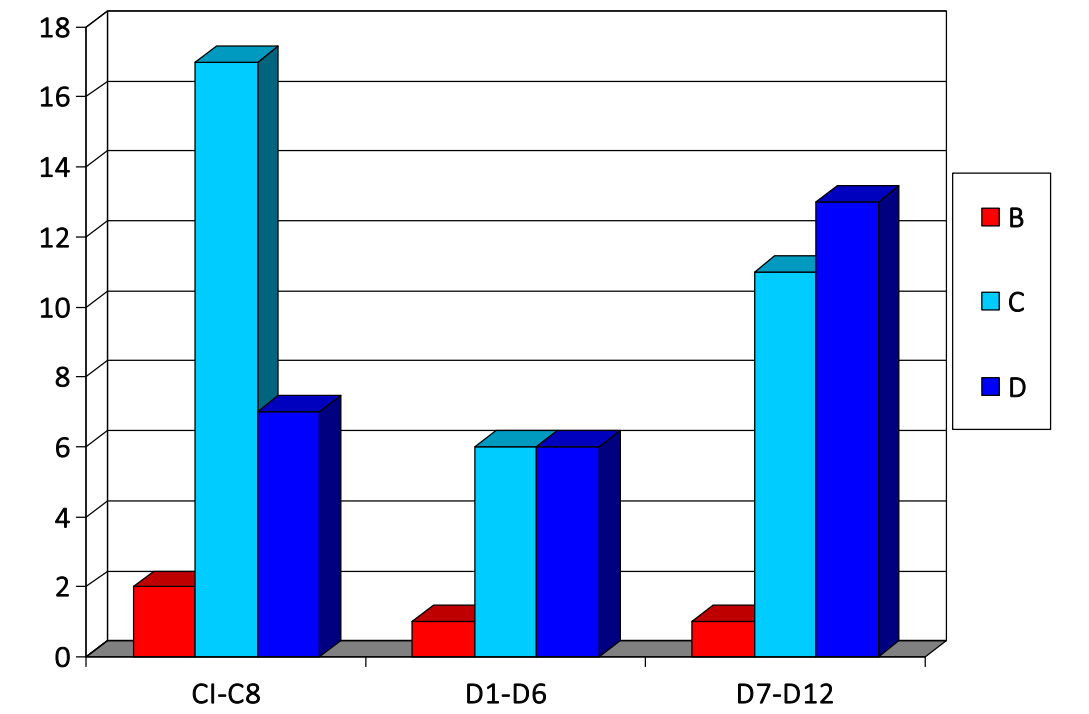

\begin{tabular}{|c|c|c|c|c|}
\hline Before & After treatment & Mean & Std. Deviation & $p$ \\
\hline Pair 1 & LEMS - f_LEMS & $-8,10938$ & 6,17244 & ,000 \\
\hline Pair 2 & WISCIII - fWISCIII & $-6,66667$ & 5,97116 & ,000 \\
\hline Pair 3 & $10 m-f 10 m$ & 2,79538 & 36,87551 & ,702 \\
\hline Pair 4 & seis - $f_{-}$seis & $-56,50333$ & 73,74744 & ,001 \\
\hline Pair 5 & upandgo - __upango $_{2}$ & $-6,91667$ & 47,58506 & ,736 \\
\hline Pair 6 & i_FIM - f_FIM & $-17,12727$ & 14,64399 & ,000 \\
\hline Pair 7 & FIM_I - f_FIM_I & $-3,43636$ & 3,11961 & ,000 \\
\hline Pair 8 & SCIM-f_SCIM & $-17,13333$ & 14,84863 & ,000 \\
\hline Pair 9 & SCIM_mov - f_SCIM_mov & $-7,46667$ & 6,80441 & ,000 \\
\hline Pair 10 & Euroq100 - f_Euroq100 & $-11,692$ & 25,510 & 007 \\
\hline
\end{tabular}

\begin{tabular}{ccccc} 
& Etiology & N & Mean (SD) & p \\
\hline \multirow{2}{*}{ LEMS } & Traumatic & 18 & $6.1(7.0)$ & \\
& Medical & 45 & $8.7(6.0)$ & $>0.05$ \\
\multirow{2}{*}{ WISCI II } & Traumatic & 15 & $7.6(6.3)$ & \\
& Medical & 37 & $8.4(5.4)$ & $>0.05$ \\
& Traumatic & 12 & $67.5(73.5)$ & 0.034 \\
6 min & Medical & 36 & $128.7(121.6)$ & \\
& Traumatic & 7 & $34.7(35.0)$ & $>0.05$ \\
Up-go & Medical & 22 & $34.9(29.6)$ & \\
& Traumatic & 15 & $14.3(12.0)$ & 0.04 \\
FIM & Medical & 43 & $16.8(15.7)$ & \\
& Traumatic & 13 & $11.687 .9)$ & 0.02 \\
SCIM & Medical & 35 & $17.5(16.6)$ & \\
& Traumatic & 12 & $.71(.2)$ & $>0.05$ \\
EQ5D & Medical & 34 & $.64(.2)$ &
\end{tabular}

\begin{tabular}{ccccc} 
& Evolution time & N & Mean (SD) & p \\
\hline \multirow{2}{*}{ LEMS } & $>90$ días & 37 & $7.4(7.5)$ & $>0.05$ \\
& $<90$ días & 26 & $8.4(4.0)$ & \\
\multirow{2}{*}{ WISCI II } & $>90$ días & 31 & $5.9(4.4)$ & 0.01 \\
& $<90$ días & 22 & $11.1(5.7)$ & \\
\multirow{2}{*}{6 min } & $>90$ días & 27 & $68.8(93.8)$ & 0.01 \\
& $<90$ días & 20 & $174.4(115.5)$ & \\
\multirow{2}{*}{ Up-go } & $>90$ días & 12 & $24.3(29.1)$ & $>0.05$ \\
& $<90$ días & 16 & $42.1(30.7)$ & \\
FIM & $>90$ días & 31 & $12.6(11.7)$ & 0.01 \\
& $>90$ días & 28 & $20.1(16.7)$ & \\
SCIM & $>90$ días & 23 & $12.0(12.9)$ & $>0.05$ \\
& $>90$ días & 25 & $19.1(15.5)$ & \\
EQ5D & $>90$ días & 23 & $0.7(0.2)$ & $>0.05$ \\
& $<90$ días & 23 & $0.6(02)$ &
\end{tabular}

\section{Conclusions.-}

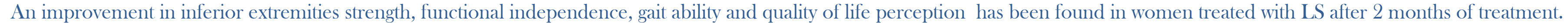
Every analysed variable has improved despite patient age.

Better results for daily life activities independence and gait resistance can be obtained in patients with non-traumatic SCI.

Patients that begun treatment less than 3 months after lesion obtained better results in the speed test and in FIM-L. 